damit im Zusammenhang stehende Anforderungen handeln, die selbstständig nicht kompensiert werden können und die auf Dauer bestehen. Auch im Krankenhaus muss künftig der tatsächliche Pflegebedarf eines Patienten im Vordergrund stehen. Dieser lässt sich auch hier am besten über den Grad der Selbstständigkeit eines Patienten ermitteln. Dafür gibt es bereits passende Instrumente.

\section{DRG-Kalkulationen erweitern}

Dabei gilt es, die kognitiven und körperlichen Funktionsfähigkeiten als Pflegebedarfsfaktoren in die DRG-Kalkulation einfließen zu lassen. Maßgeblich kann dadurch der Grad der Selbstständigkeit und der damit verbundene Pflegeaufwand abgebildet werden. Nötig ist hierfür lediglich die stärkere Einbindung von Assessmentinstrumenten in die DRGSystematik. Diese würden darüber hinaus für die Weiterentwicklung der Qualitätsindikatoren zur Verfügung stehen.

\section{Nursing Related Groups sind ungeeignet}

Nicht zielführend ist dagegen die Diskussion um eine Einführung von Nursing Related Groups (NRG). Die NRGs stellen nichts weniger als die Ausweitung der PKMS-Systematik auf alle Patienten dar. Dies ist aufgrund der fachlichen Mängel, der Prüfanfälligkeit und der Dokumentationsaufwände nicht die Lösung, sondern bindet mehr Pflegezeit für Dokumentation und macht den Pflegeberuf weiter unattraktiv.

Der Deutsche Pflegerat hat seinen Vorschlag für die Pflegebedarfsfaktoren, mit deren Hilfe die pflegerischen Leistungen im DRG-System besser abgebildet werden, im Rahmen des DRG-Forums 2015 vorgestellt. Jetzt muss es darum gehen, unser sinnvolles Instrument gemeinschaftlich weiter auszubauen. Die Pflege muss unbürokratisch und leistungsgerecht im Finanzierungssystem der Krankenhäuser abgebildet werden. Die Integration von Pflegebedarfsfaktoren ist dafür der richtige Ansatz.

\section{Dr. Patrick Jahn}

Leiter der DPR-Fachkommission DRG

\title{
Pflegereform bietet keine Antwort auf den Fachkräftemangel
}

— In mehreren Interviews hat der Präsident des Deutschen Pflegerats (DPR), Andreas Westerfellhaus, ausführlich Rede und Antwort zum Beschluss des Bundeskabinetts gestanden, das Zweite Pflegestärkungsgesetz auf den gesetzgeberischen Weg zu bringen. Im Folgenden finden Sie hierzu Auszüge aus den Gesprächen. Gesprochen haben mit Andreas Westerfellhaus Tobias Armbrüster vom Deutschlandfunk und Martin Steinhage vom Deutschlandradio Kultur.

Deutschlandfunk. Hier heißt es: In der Theorie gut, in der Praxis mit Problemen behaftet - so lautet das Urteil von Andreas Westerfellhaus über die Pflegereform. Die Regierung bleibe eine Antwort auf den Mangel an Pflegekräften schuldig.

Westerfellhaus wies darauf hin, dass auch in der Berufsgruppe der Pflegekräfte eine demografische Entwicklung stattfinde und viele von ihnen in den kommenden Jahren in Rente gingen. Wenn sich die Regierung nicht ganz schnell um Nachwuchs bemühe, werde die Pflegereform in ihren Ansätzen steckenbleiben. Er fordert eine bessere Bezahlung der Pflegekräfte und deutschlandweit einheitliche Tarife.

An die Politik appelliert Westerfellhaus, der Gesundheitspolitik in Deutschland denselben Stellenwert einzuräumen wie etwa der Energie- und Umweltpolitik. Aber auch die Menschen selbst müssten zur Kenntnis nehmen, dass jeder irgendwann pflegebedürftig werde. In diesem Punkt habe die Gesellschaft lange Zeit aktiv weg geschaut, mahnte der DPR-Präsident im Deutschlandfunk.

Deutschlandradio Kultur. Hier sagte Andreas Westerfellhaus auf die Frage, ob es für die Abdeckung des mit der Pflegereform einhergehenden Mehraufwandes genügend qualifiziertes Personal gebe: „Das ist natürlich genau das große Problem. Und das ist letzt- endlich auch jetzt der Kritikpunkt an der Pflegereform.

Man muss sich darüber im Klaren sein, dass die Frage nicht beantwortet wird: Mit wem wollen wir diese intensive Leistung letztendlich erbringen? Wenn ich einen Vorwurf laut werden lassen muss, dann ist es der, dass man sich nicht gleichzeitig damit beschäftigt: Wie wollen wir eigentlich in der Zukunft mit dem bestehenden Fachkräftemangel umgehen? Und wer bitte soll diese Leistungen erbringen? Wenn wir diese Frage aus der Politik nicht beantwortet bekommen, dann ist die Enttäuschung über diese Reform vorprogrammiert". Die Reform bleibe ein Papiertiger, sofern sich an der so genannten Minutenpflege nichts ändere, sagte der DPR-Präsident weiter: „Wenn ich Menschen erkläre, sie müssen für eine zukünftige Leistung mehr Geld bezahlen über die Pflegeversicherung und sie haben einen Rechtsanspruch darauf, auf eine andere Form von Einstufung und auch auf Leistung ihnen dann allerdings erklären muss, dass das Personal leider Gottes dafür fehlt, dann ist das aus meiner Sicht eine Bankrotterklärung".

Nach wie vor habe Deutschland einen Pflegenotstand, an dem sich seit Jahren nichts geändert habe, sagte Westerfellhaus. Dieser sei in den letzten Jahren dramatischer geworden. Der DPR-Präsident weiter: „Meine Kolleginnen und Kollegen können nicht mehr! Es ist nicht nur Rot, es ist Dunkelrot und es ist fürchterlich nach Zwölf! Und ich weiß nicht, warum wir mit dieser Politik oder mit diesem Teil der Pflegepolitik ein solches Nischendasein führen". Die Gesundheits- und Pflegepolitik benötige einen höheren Stellenwert, sagte Westerfellhaus abschließend.

Quellen: www.deutschlandfunk.de und www.deutschlandradiokultur.de 